\title{
Substituent Control of Tribofilm Growth under Mechanochemical Conditions
}

Jie Zhang, James P. Ewen* and Hugh A. Spikes*

Department of Mechanical Engineering, Imperial College London, South Kensington Campus, London SW7 2AZ, U.K.

Corresponding Authors

*E-mail: j.ewen@imperial.ac.uk (James P.Ewen); h.spikes@imperial.ac.uk (Hugh A. Spikes) 


\begin{abstract}
Lubricant additives that reduce wear by forming protective tribofilms on sliding surfaces are crucial to maintaining the efficient and reliable operation of many engineering systems. The most important of these additives, zinc dialkyldithiophosphate (ZDDP), has been in use for almost a century; however, several aspects of the physicochemical mechanisms through which it reduces wear remain unclear. While changes to the molecular structure of ZDDP are known to affect tribofilm formation and antiwear performance, the underlying mechanisms are not well understood. Here, we show using macroscale tribometer experiments under well-defined temperature and stress conditions, how the ZDDP tribofilm formation rate on steel from a highfriction base oil can be controlled by tailoring the additive's alkyl substituents. Our results suggest that the chain-length, branching and presence of cycloaliphatic groups can affect the packing density, steric hindrance, and stress transmission, leading to large differences in the temperatureand stress-dependencies of the tribofilm formation rate. These changes can be successfully explained using the Bell model; a simple modification of the Arrhenius equation commonly used to model the kinetics of mechanochemical processes. Using this model, large differences in the activation energy, pre-exponential factor, and activation volume for the various ZDDPs studied become apparent. We expect these results to be useful both for the development of highperformance lubricant additives for specific applications and for improving macroscale tribology models that consider the effects of growing tribofilms.
\end{abstract}




\section{INTRODUCTION}

The development of more sustainable lubricants with tailored tribological performance is a crucial activity in the pursuit of net zero greenhouse gas emissions. ${ }^{1}$ Modern lubricants consist of a mixture of base oils, which are most often nonpolar hydrocarbons, blended with several different types of additives, which can be polar organic molecules, polymers, or organometallic compounds. ${ }^{2}$ Probably the most important of these additives is zinc dialkyldithiophosphate (ZDDP), which was originally developed as an antioxidant, but is now used primarily for its remarkable antiwear performance. ${ }^{3}$ ZDDP reduces wear by forming protective 'tribofilms' on rubbing steel surfaces. ${ }^{3}$ Despite decades of research and development to find a more environmentally-friendly alternative, ${ }^{4}$ ZDDP remains ubiquitous in engine lubricants. To rationally design antiwear additives with improved performance, a molecular-level understanding of the tribofilm formation and wear-reduction mechanisms of ZDDP are first required. ${ }^{2}$ The tribofilms formed by ZDDP on steel substrates are mainly composed of zinc and iron polyphosphates. ${ }^{3}$ These are formed by nucleophilic substitution reactions at the $\mathrm{P}$ atoms, which result in the formation of the $\mathrm{P}-\mathrm{O}-\mathrm{P}$ chains. ${ }^{5}$ This reaction can be accelerated by removing the alkyl or aryl groups through $\mathrm{C}-\mathrm{O}$ cleavage. ${ }^{6}$ Therefore, ZDDPs containing different alkyl and aryl groups show greatly varying tribofilm growth and antiwear performance; ${ }^{7-9}$ however, the underlying molecular mechanisms for these differences in macroscale behavior remain unclear.

In recent years, the mechanochemical aspects of ZDDP tribofilm formation have attracted widespread attention. Atomic force microscopy (AFM) experiments showed that ZDDP tribofilm formation rate increases exponentially with temperature and stress. ${ }^{10}$ This discovery indicated that ZDDP tribofilm formation is a stress-augmented, thermally activated (SATA) process. ${ }^{11}$ The rate 
constant, $k$, for a SATA process can be given by a modified form of the Arrhenius equation, which was originally proposed by Evans and Polanyi, ${ }^{12}$ but is now usually known as the Bell model: ${ }^{13}$

$k=A \exp \left(-\frac{E_{a}-\sigma \Delta V^{*}}{k_{B} T}\right)$

where $A$ is the pre-exponential factor, $E_{a}$ is the activation energy, $\sigma$ is the applied stress, $\Delta V^{*}$ is the activation volume, and $k_{B}$ is the Boltzmann constant. ${ }^{14}$ Although both the normal stress ${ }^{10}$ and the shear stress ${ }^{15,16}$ have been used in this equation for ZDDP tribofilm formation, recent experiments have demonstrated that the latter controls the reaction rate in macroscale experiments. ${ }^{15,16}$ Tribometer experiments at different scales have suggested that ZDDP tribofilm formation follows either fractional-order ${ }^{10,17}$ or zero-order ${ }^{10,16}$ kinetics, indicating that ZDDP adsorbs onto steel surfaces before it dissociates and forms a tribofilm.

Much faster tribofilm growth has been observed for ZDDPs with secondary alkyl substituents compared to primary ones in macroscale tribometer experiments under both mixed/boundary (load mostly supported by the solid asperities $)^{8}$ and full-film elastohydrodynamic lubrication (EHL) (load supported by the pressurized lubricating fluid) ${ }^{16}$ conditions, which has been rationalized by the greater stability of the carbocation formed during $\mathrm{C}-\mathrm{O}$ cleavage in the former. ${ }^{18}$ Since the initial $\mathrm{C}-\mathrm{O}$ cleavage reaction is the rate-determining step for ZDDP tribofilm formation, ${ }^{16}$ this bond is likely to be the key mechanophore for this process. Although shear stress controls the rate of tribofilm formation at the macroscale, ${ }^{16}$ at the molecular level it is likely that the rate of $\mathrm{C}-\mathrm{O}$ cleavage is accelerated by tensile stress. ${ }^{13}$ 
The structure and position of the chemical groups surrounding the mechanophore is known to influence the reactivity of mechanochemical processes. For example, in polymer mechanochemistry, longer chains are known to lead to enhanced reactivity in both bulk liquid flows ${ }^{19}$ and solid grinding experiments. ${ }^{20}$ Single-molecule force spectroscopy experiments have shown how the 'lever-arm effect' can enhance mechanochemical reactivity by polymer backbones acting as phenomenological levers that impose greater mechanical distortion on the mechanophore. ${ }^{21}$ Cyclopropane-based polymers containing $E$-alkenes showed activation lengths (force-dependent equivalent of the stress-dependent activation volume) that were a third larger than those containing $Z$-alkenes. ${ }^{22}$ Substituent effects can differ depending on the reaction pathway; for example, cyclobutene-based polymers containing trans-alkyl handles provided more mechanical leverage than trans-ester handles in conrotatory reactions, whereas cis-ester handles gave more mechanical leverage than $c i s$-alkyl handles in disrotatory reactions. ${ }^{23}$ The enhanced mechanical leverage of dialkyl relative to diester attachments was consistent with quantum chemical calculations reported previously on trans-cyclobutene derivatives. ${ }^{24}$ In similar polymers, replacing a methylene in the pulling attachment with a phenyl group dropped the force necessary to achieve a given rate constant in SMFS experiments by a factor of three, which was attributed to a combination of electronic stabilization and mechanical leverage effects. ${ }^{25}$ More conventional substituent effects that can be explained through linear free energy relationships, ${ }^{26}$ have also been identified using SMFS. For example, spiropyran-derived polymers containing more electronwithdrawing substituents in the para position to the labile spirocyclic $\mathrm{C}-\mathrm{O}$ bond required less force to break. ${ }^{27}$ Substituent effects have also been predicted for small molecules from quantum chemical calculations, ${ }^{28,29}$ however, to our knowledge, substituent effects, such as the lever arm 
effect, are yet to be detected experimentally for molecular or organometallic systems, such as ZDDP.

Here, we investigate the mechanochemical tribofilm formation of ZDDPs containing several different aliphatic substituents on steel surfaces in a ball-on-disk tribometer under full-film EHL conditions. The methods used here are similar to those described by Zhang et al. ${ }^{16}$, but employing different base oils and additives. In these experiments, the temperature and shear stress conditions can be carefully controlled, ${ }^{30}$ which is not the case in mixed/boundary lubrication conditions as present in most mechanochemistry apparatus, such as ball mills. ${ }^{31}$ This is because the stress is applied through confined base oil molecules rather than randomly contacting solid asperities. ${ }^{15,16}$ This approach also avoids tribofilm wear ${ }^{32}$ and other potential drivers of tribofilm growth, such as frictional heating, triboemission, and generation of catalytic surfaces. ${ }^{33}$ For measurable tribofilm formation to occur under these conditions, large stresses are required, which can be achieved by using either hard surfaces ${ }^{15}$ or very high applied loads. ${ }^{16}$ Moreover, base oils that provide high shear stress such as traction fluids with bulky cycloaliphatic groups ${ }^{15,16}$ or polymers with pendant methyl groups ${ }^{16}$ are required. High additive-surface affinity is also needed to form ZDDP tribofilms under these conditions. ${ }^{34}$ Unlike previous studies using lower-purity commercial additives, ${ }^{10,15-17}$ the ZDDPs used here contain a wide range of well-defined alkyl groups to provide a more detailed understanding of the substituent effects on tribofilm growth under mechanochemical conditions. Using the Bell model, ${ }^{13}$ large differences in the activation energy, pre-exponential factor, and activation volume for the various ZDDPs studied become apparent. We anticipate that these results will be useful both for the development of high-performance 
antiwear additives for specific applications ${ }^{2}$ and for improving the accuracy of macroscale tribology models that include the effects of tribofilm growth. ${ }^{35-38}$

\section{METHODOLOGY}

\section{Materials}

All the tribometer experiments used through-hardened AISI 52100 steel balls and disks from PCS Instruments (Acton, UK). For the Hertz pressure and shear stress calculations, ${ }^{16}$ the elastic modulus was taken as $207 \mathrm{GPa}$ and 0.3 was used for the Poisson's ratio. ${ }^{39}$ The ball had a rootmean-square (RMS) roughness, $\mathrm{R}_{\mathrm{q}}=8.5 \mathrm{~nm}$, while for the disk, $\mathrm{R}_{\mathrm{q}}=6.0 \mathrm{~nm}$. The steel ball and disc were soaked in toluene for at least 24 hours before a test and then ultrasonically cleaned in toluene, followed by Analar acetone, before being mounted in the tribometer.

We studied seven ZDDPs with different alkyl groups; four primary (oct-1-yl, 2-ethylhexyl, 2cyclohexylethyl, and dodec-1-yl) and three secondary (oct-2-yl, but-2-yl, and 4-methylpent-2-yl). The ZDDPs were synthesized by Afton Chemical Corporation (Richmond, VA). The ZDDPs were synthesized from single high-purity ( $\geq 98 \%$ ) alcohols, purchased from MilliporeSigma (St. Louis, MO) and used without further purification. This ensured that high-purity ZDDPs with well-defined chemical structures were tested. ${ }^{9}$ Our ${ }^{1} \mathrm{H}-\mathrm{NMR}$ and ${ }^{13} \mathrm{C}-\mathrm{NMR}$ analysis confirmed that six of the ZDDPs contained only the expected alkyl group, while the oct-2-yl ZDDP contained 98 mol.\% oct-2-yl and 2 mol.\% oct-1-yl. Previous studies have suggested that negligible alkyl rearrangement occurs during ZDDP synthesis. ${ }^{18}$ The ZDDPs were mostly ( $>80$ mol.\%) in their neutral form, but they did contain a small amount of the basic form. ${ }^{40}$ Previous tribology studies have shown that the two forms behave quite similarly. ${ }^{3}$ All of the solutions contained a total ZDDP concentration 
of $800 \mathrm{ppm}$ of phosphorus, which is similar to the treat rate used in commercial lubricant formulations. $^{16}$

Strong additive adsorption is important to tribofilm formation under full-film EHL conditions. ${ }^{34}$ Previous powder adsorption experiments have shown very similar adsorption behavior for linear primary ( $n$-butyl), methyl-branched primary ( $i$-butyl), and secondary ( $s$-butyl) ZDDPs from $n$ hexadecane onto iron oxide surfaces. ${ }^{41}$ Therefore, for most of the ZDDPs studied here, the adsorption behavior is not expected to significantly affect the tribofilm formation rate on steel. However, for the ZDDPs containing longer branches ${ }^{42}$ (2-ethylhexyl) and particularly the bulky cycloaliphatic substituents $^{43}$ (2-cyclohexylethyl), SAMs with lower packing densities are expected to form, which may affect their mechanochemical reactivity.

Indopol H-8, provided by INEOS (London, UK), was used as the base oil for of the tribometer experiments in this study. This is a polybutene oligomer with an average molecular weight, $\mathrm{M}_{\mathrm{n}}=$ $490 \mathrm{~g} \mathrm{~mol}^{-1}$ (degree of polymerization $\approx 4$ ) and a polydispersity index, $\mathrm{M}_{\mathrm{w}} / \mathrm{M}_{\mathrm{n}}=1.85$. EHL fluid film thickness is determined by the lubricant viscosity at the inlet, where pressure is relatively low ( $\sim 100 \mathrm{MPa})$. At ambient pressure, Indopol H-8 has a kinematic viscosity of $15 \mathrm{cSt}$ at $100{ }^{\circ} \mathrm{C}$, which is similar to the low-molecular weight polyisobutene $\left(14 \mathrm{cSt}\right.$ at $\left.100^{\circ} \mathrm{C}\right)$, but much higher than the poly- $\alpha$-olefin base oil $\left(6 \mathrm{cSt}\right.$ at $\left.100{ }^{\circ} \mathrm{C}\right)$ and the DM2H traction fluid $\left(4 \mathrm{cSt}\right.$ at $\left.100{ }^{\circ} \mathrm{C}\right)$ that we have used previously. ${ }^{16}$ The high viscosity of Indopol H-8 ensured that thick fluid EHL films $(h>50$ $\mathrm{nm}$ ) were formed under all of the conditions used here. 

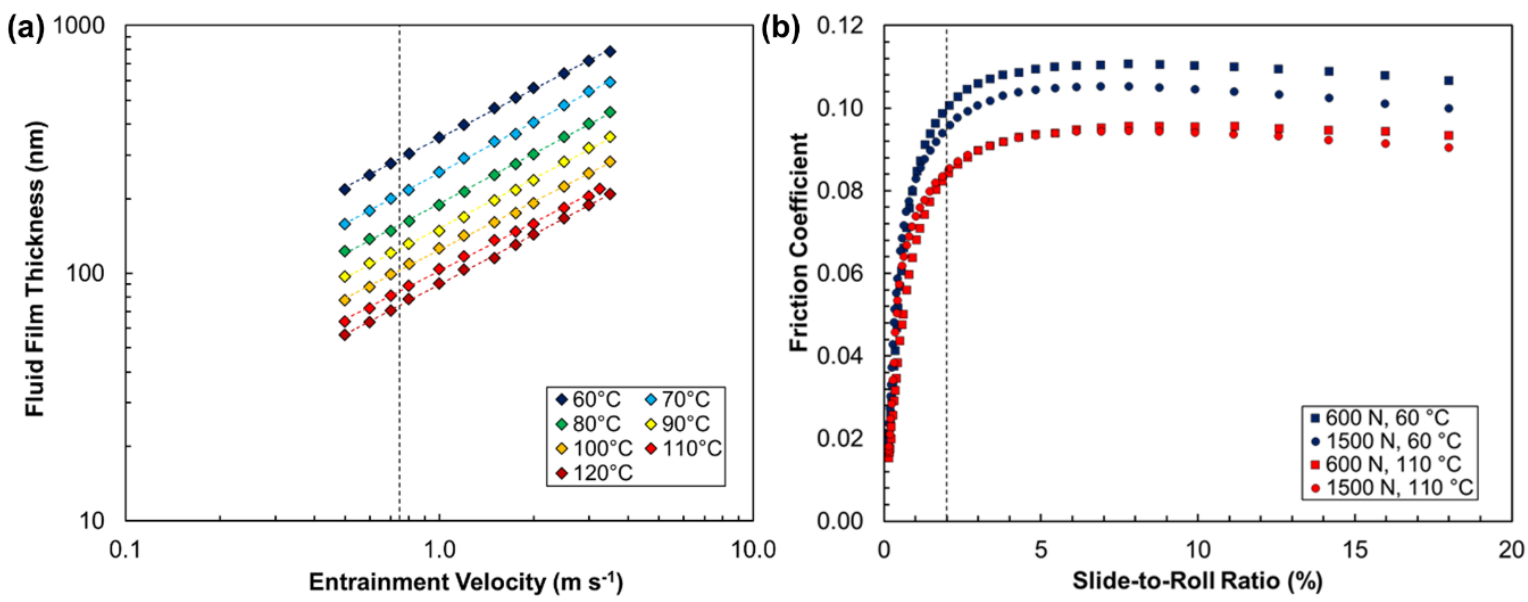

Figure 1. (a) Variation in the fluid film thickness with entrainment velocity for Indopol H-8 at different temperatures $\left(60-120^{\circ} \mathrm{C}\right)$ and a constant load $(20 \mathrm{~N})$. The vertical black line indicates the entrainment velocity used in the tribofilm growth experiments $\left(0.75 \mathrm{~m} \mathrm{~s}^{-1}\right)$. (b) Variation in the friction coefficient with slide-to-roll ratio at different for Indopol H-8 at the different temperatures $\left(60-110^{\circ} \mathrm{C}\right)$ and loads $(600-1500 \mathrm{~N})$ used in the tribofilm growth experiments. The vertical black line indicates the slide-to-roll ratio used in the tribofilm growth experiments $(2 \%)$.

The fluid film thickness of Indopol H-8 was measured in an EHD2 rig from PCS Instruments (Acton, UK) using ultra-thin film interferometry, ${ }^{44}$ as shown in Figure 1a. This technique requires the disk to be optically transparent, so a steel/glass contact, rather than steel/steel as used in the tribometer experiments, was employed. The measured fluid film thickness values were corrected using the equation due to Chittenden et al. $^{45}$ to account for the difference in reduced elastic modulus between glass and steel, as well as the large loads $(W=600-1500 \mathrm{~N})$ applied in the subsequent tribofilm growth experiments. The severity of the asperity contact can be quantified by the $\lambda$ ratio, which is the ratio of the EHL film thickness to the composite RMS surface roughness, $R_{q}$. The $R_{q}$ of the steel/steel ball-on-disk combination was $10.4 \mathrm{~nm}$, so the $\lambda$ ratios in this study 
varied between approximately $5\left(120^{\circ} \mathrm{C}\right)$ and $21\left(60^{\circ} \mathrm{C}\right)$. Since $\lambda \geq 5$, full-film EHL conditions with negligible direct asperity contact can therefore be assumed in all the tribofilm growth experiments. ${ }^{46}$ This ensures that the stress conditions are well controlled ${ }^{30}$ and mitigates tribofilm wear $^{8}$ and competing tribochemical phenomena. ${ }^{33}$

Unlike EHL film thickness, EHL friction is determined by viscosity in the central region of the contact, where the pressure is very high $(>1 \mathrm{GPa}) .{ }^{30}$ Whereas lubricant base oils designed to give low friction such as poly- $\alpha$-olefin have low pressure-viscosity coefficients $(\alpha)$, polymers (e.g. polyisobutene) and traction fluids (e.g. 2,3-dimethyl-2-[(3-methylbicyclo[2.2.1]hept-2yl)methyl]-bicyclo[2.2.1]heptane) have high $\alpha$ values. ${ }^{16}$ Although the $\alpha$ value has not been reported for Indopol H-8, it is expected to be similar to the value measured for high-molecular weight polybutenes $\left(\alpha \approx 30 \mathrm{GPa}^{-1}\right) .{ }^{47}$ Therefore, EHL friction (and thus shear stress) is likely to be sufficiently high to promote tribofilm growth on the steel surfaces in the accessible temperature and load range. ${ }^{16}$ To quantify the shear stress conditions under which the tribofilms were grown, we measured this quantity in ball-on-disk tribometer experiments using the Extreme-pressure Traction Machine (ETM) from PCS Instruments (Acton, UK). Figure $1 \mathrm{~b}$ shows that, in the absence of ZDDP, the friction coefficient of Indopol H-8 slightly increases with increasing load and decreases with increasing temperature. The EHL friction was also measured during the tribofilm growth experiments to ensure that this did not change due to the presence of the tribofilm. ${ }^{16}$ To convert the measured EHL friction coefficients to maximum shear stresses, they were multiplied by the maximum Hertz pressure $\left(P_{\max }=2.5-3.5 \mathrm{GPa}\right)$ at the corresponding load. This approach provides the stress conditions in the central region of the ball-on-disk contact, where the tribofilm is formed. ${ }^{16}$ 


\section{Methods}

For the tribofilm growth measurements, we performed ball-on-disk tribometer experiments using the ETM (schematic shown in Figure S1). ${ }^{16}$ We used the same ratio of the sliding velocity to the entrainment velocity, known as the slide-to-roll ratio $(S R R=2 \%)$ as in our previous tribofilm growth study. ${ }^{16}$ Since Indopol H-8 has a higher viscosity than the traction fluid DM2H used in our previous study, ${ }^{16}$ a sufficiently thick fluid film could be generated with a lower entrainment velocity $\left(U=0.75 \mathrm{~m} \mathrm{~s}^{-1}\right)$, and the sliding velocity was reduced accordingly $\left(u_{s}=0.015 \mathrm{~m} \mathrm{~s}^{-1}\right)$ to maintain the same $S R R$. This approach reduced frictional heating compared to the conditions used in our previous study. ${ }^{16}$ The amount of frictional heating during the tribofilm growth experiments was estimated using the Archard equation, ${ }^{48}$ employing the recently measured thermal conductivity of through-hardened AISI 52100 steel. $^{49}$ The rise in surface temperature in the contact due to frictional heating is expected to increase slightly with load from $2.6^{\circ} \mathrm{C}$ at $600 \mathrm{~N}$ to $4.8^{\circ} \mathrm{C}$ at $1500 \mathrm{~N}$. Thus, the significant increase in tribofilm formation rate at higher loads observed below cannot be explained by frictional heating, which confirms mechanochemical acceleration.

During each test, the lubricant was added to the test chamber and the system was heated with the ball and disc rotating but not in contact. Once the desired lubricant temperature $\left(T=60-120{ }^{\circ} \mathrm{C}\right)$ was reached, a short running-in procedure was carried out at conditions of $U=0.1 \mathrm{~m} \mathrm{~s}^{-1}, S R R=$ $50 \%$, and $W=100 \mathrm{~N}$ load for 1 minute. The short running-in stage neither resulted in any measurable change in surface roughness of the specimens nor was it essential for subsequent tribofilm formation; however, it did improve the repeatability of the tribofilm formation tests, possibly by removing any adsorbed contaminants on the test specimens by rubbing. ${ }^{16}$ The contact 
was then unloaded and $U$ increased to $0.75 \mathrm{~m} \mathrm{~s}^{-1}$ with $\mathrm{SRR}=2 \%$. The target load $(W=600-1500$ N) was then applied and an extended test carried out under full-film EHL conditions. The accessible loads correspond to a maximum Hertz pressure range of 2.5-3.5 GPa in the steel/steel ball-on-disk contact. ${ }^{16}$
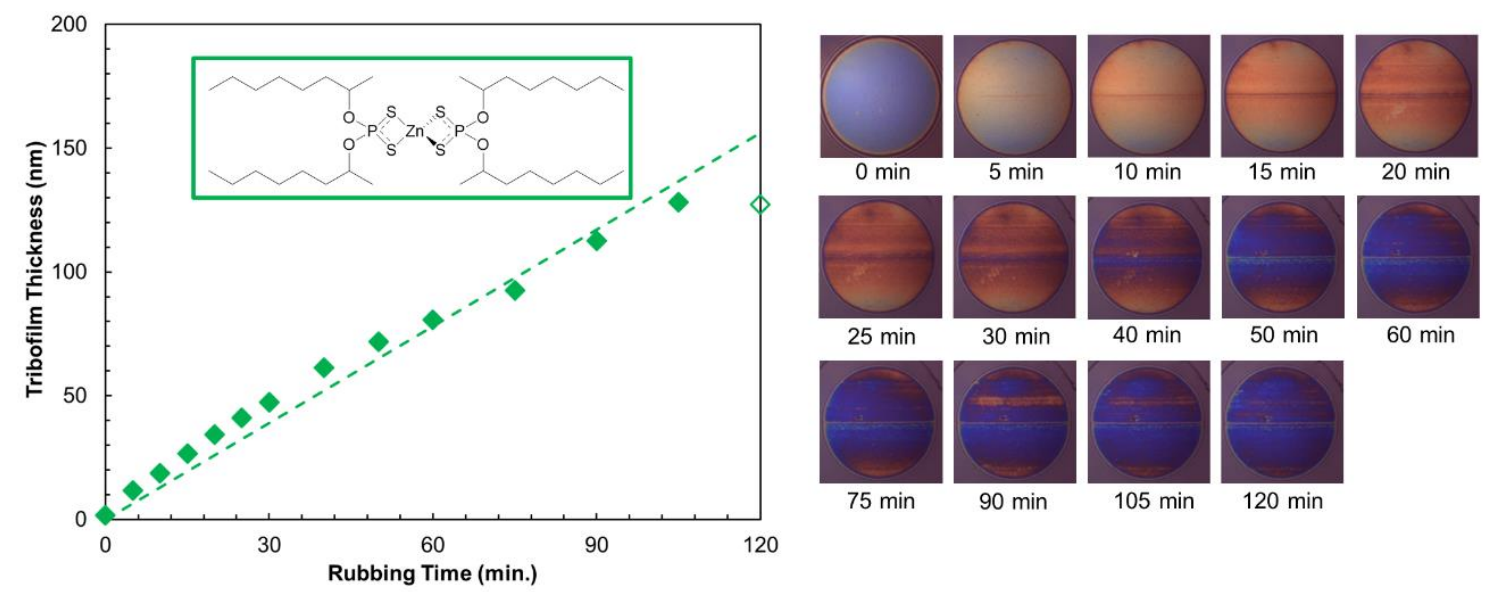

$60 \mathrm{~min}$

Figure 2. Representative example (oct-2-yl ZDDP at $80{ }^{\circ} \mathrm{C}$ and $1000 \mathrm{~N}$ ) of the tribofilm thickness growth over time, measured from the SLIM images shown on the right. Dashed lines are the linear fits used to calculate the rates assuming zero-order kinetics; only the filled symbols are used for the fitting.

The tribofilm thickness was determined every 5-15 minutes using spacer-layer imaging (SLIM). ${ }^{50}$ During the tests, the ball was raised from the disk, motion was halted, and the ball was loaded upward, within the test chamber, against a coated glass window with a load of $100 \mathrm{~N}$. White light was then shone into the resulting contact, where some is reflected by a semi-reflective coating on the underside of the glass. The remaining light passes through a silica spacer layer and the tribofilm 
formed on the ball, before being reflected back from the steel substrate. The two beams undergo optical interference depending on their optical path difference, which corresponds to the tribofilm thickness. An interference image was captured as a red-green-blue (RGB) pixel map using a highresolution camera. A calibration chart of RGB color versus optical path difference obtained using ultra-thin film interferometry ${ }^{44}$ was then used to convert this RGB pixel map to a tribofilm thickness map. A refractive index of 1.6 was assumed for the tribofilm. ${ }^{8}$ A representative set of SLIM images and the corresponding tribofilm thicknesses are shown in Figure 2.

\section{RESULTS AND DISCUSSION}

Figure 3 shows how the tribofilm thickness varies with rubbing time for the different ZDDP solutions in Indopol-H8 at the same concentration (800 ppm P), temperature $\left(80^{\circ} \mathrm{C}\right)$ and load $(1000 \mathrm{~N})$ condition. At the start of the tests, the tribofilm formation rate is approximately linear in all cases, which is indicative of zero-order kinetics. ${ }^{16}$ The tribofilm formation rates are much higher for all the secondary ZDDPs than the primary ones, which is consistent with previous tribometer experiments with commercial ZDDPs under mixed/boundary ${ }^{8}$ and full-film EHL conditions. ${ }^{16}$ The three secondary ZDDPs and one of the primary ZDDPs (oct-1-yl) level out at a maximum tribofilm thickness of between $50-150 \mathrm{~nm}$, while the other primary ZDDPs continue to increase linearly over the duration of the experiments. A similar range of maximum tribofilm thicknesses have been observed in previous experiments. ${ }^{8,10,16}$ This levelling-off behavior has been attributed to the reduction in contact stress for thicker ZDDP tribofilms due to their lower stiffness compared to the underlying steel substrate..$^{10}$ 


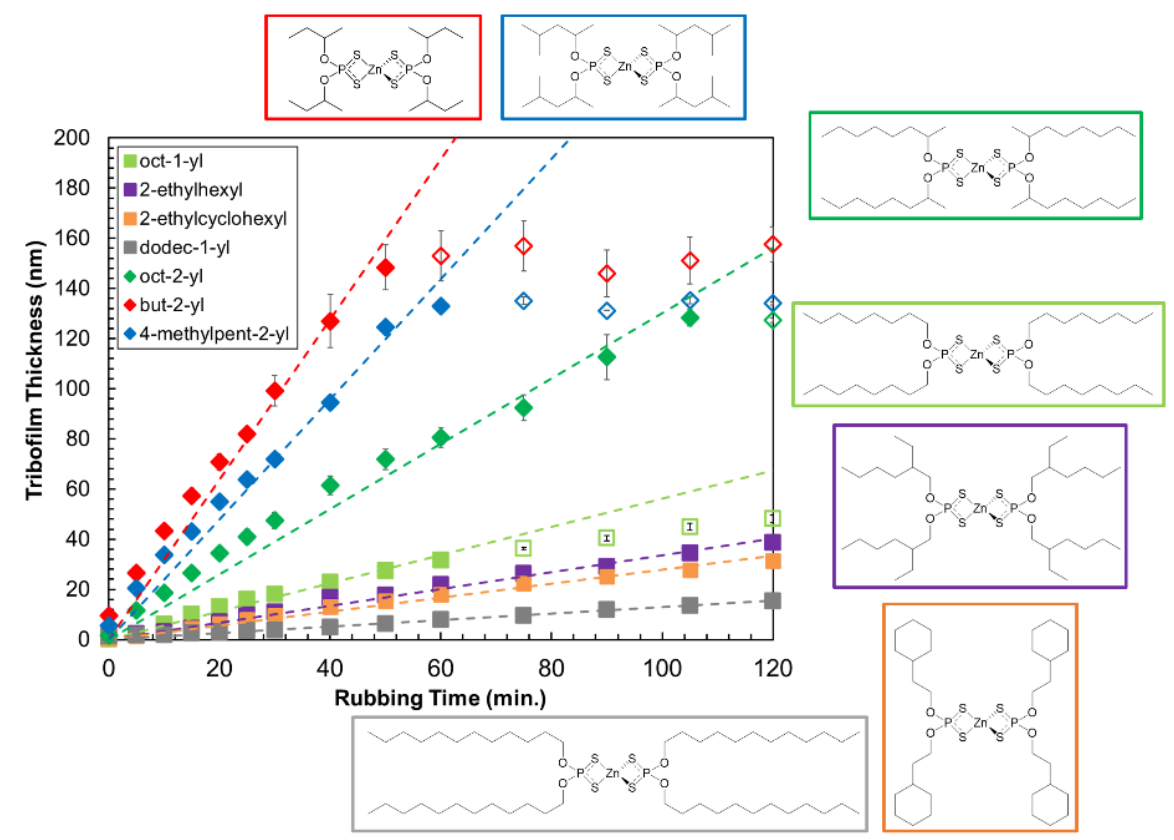

Figure 3. Variation in the tribofilm thickness with rubbing time for the primary (oct-1-yl, 2ethylhexyl, 2-cyclohexylethyl, and dodec-1-yl) and secondary (oct-2-yl, but-2-yl, and 4methylpent-2-yl) ZDDPs for a single temperature $\left(80^{\circ} \mathrm{C}\right)$ and load $(1000 \mathrm{~N})$ condition in steel/steel contacts. Points represent mean results from three tests, vertical bars represent one standard deviation. Dashed lines are the linear fits used to calculate the rates assuming zero-order kinetics; only the filled symbols are used for the fitting.

The differences in tribofilm formation rate with chain length, branching, and the presence of cycloaliphatic groups are more subtle. For the primary alkyl ZDDPs, the reactivity at $80{ }^{\circ} \mathrm{C}$ and load $1000 \mathrm{~N}$ increases in the order: dodec-1-yl < 2-cyclohexylethyl < 2-ethylhexyl < oct-1-yl. Comparing the linear alkyls, the ZDDP with the longer chain (dodec-1-yl) forms tribofilms much more slowly than that with shorter chains (oct-1-yl). A reduction in friction with increasing chain length has also been noted for oct-1-yl and dodec-1-yl ZDDPs under boundary lubrication conditions. ${ }^{51}$ These observations suggest that ZDDP (or its dissociation products) form self- 
assembled monolayers (SAMs) on top of the growing ZDDP tribofilm that are responsible for both the boundary friction response ${ }^{9}$ and surface reactivity. SAM formation has also been linked to the antiwear performance of ZDDPs in previous studies where molecules with larger monolayer cohesive energies gave lower wear. ${ }^{52,53}$ It has been shown that for a range of systems and processes at solid-liquid interfaces that SAMs formed from surfactants with longer alkyl chains generally show lower chemical reactivity. ${ }^{54-56}$ This is due to the formation of more densely packed, ordered monolayers $^{57}$ that are more difficult for approaching molecules to penetrate to react with the surface. This effect is likely responsible for the slower tribofilm growth of dodec-1-yl ZDDP than octyl-1-yl ZDDP, since the more densely packed monolayer hinders adsorption, decomposition, and ultimately tribofilm formation on the steel surface. For the primary ZDDPs with $\mathrm{C}_{8}$ alkyl groups, the tribofilm growth rate increases in the order: 2-cyclohexylethyl $<2$-ethylhexyl $<$ oct-1yl. This suggests that the increased steric bulk has a larger effect on the rate than the lower packing density expected for the ethyl-branched ${ }^{42}$ and particularly cyclohexyl-containing ${ }^{43}$ ZDDPs compared to the linear one. Previous studies of other systems have also shown lower reactivity for surfaces protected by branched alkyl monolayers compared to linear ones. ${ }^{42,58}$

For the secondary alkyl ZDDPs at $80{ }^{\circ} \mathrm{C}$ and $1000 \mathrm{~N}$, the reactivity increases in the order: oct-2$\mathrm{yl}<4$-methylpent-2-yl $<$ but-2-yl. The trend is similar to the primary ZDDPs in that the secondary ZDDPs with longer alkyl chains give slower tribofilm formation rates, which can again be attributed to the formation of more densely packed SAMs. The presence of methyl branches in the 4-methylpent-2-yl ZDDP seems to have a minimal effect on the tribofilm growth rate, since the rate is approximately halfway between oct-2-yl and but-2-yl, as would be expected for a linear hex-2-yl group. A similar order of tribofilm growth rate for ZDDPs with the same substituents has 
recently been reported under mixed/boundary conditions, ${ }^{9}$ where the tribofilm will be partially worn away as it grows. ${ }^{8}$

To obtain a more complete understanding of substituent effects on mechanochemical reactivity of ZDDP, we studied how the tribofilm formation rate varied with temperature $\left(60-120^{\circ} \mathrm{C}\right)$ and load $(600-1500 \mathrm{~N})$. Figure 4 shows the tribofilm growth for a representative primary (oct-1-yl) and secondary (oct-2-yl) ZDDP. Tribofilm growth is linear for both ZDDPs up to a constant thickness. The growth rate increases with both temperature and load. The growth rate is faster for the oct-2yl ZDDP than the oct-1-yl ZDDP for all the temperature-load combinations studied. Results for the other primary and secondary ZDDPs are shown in Figure S2 and Figure S3, respectively. 

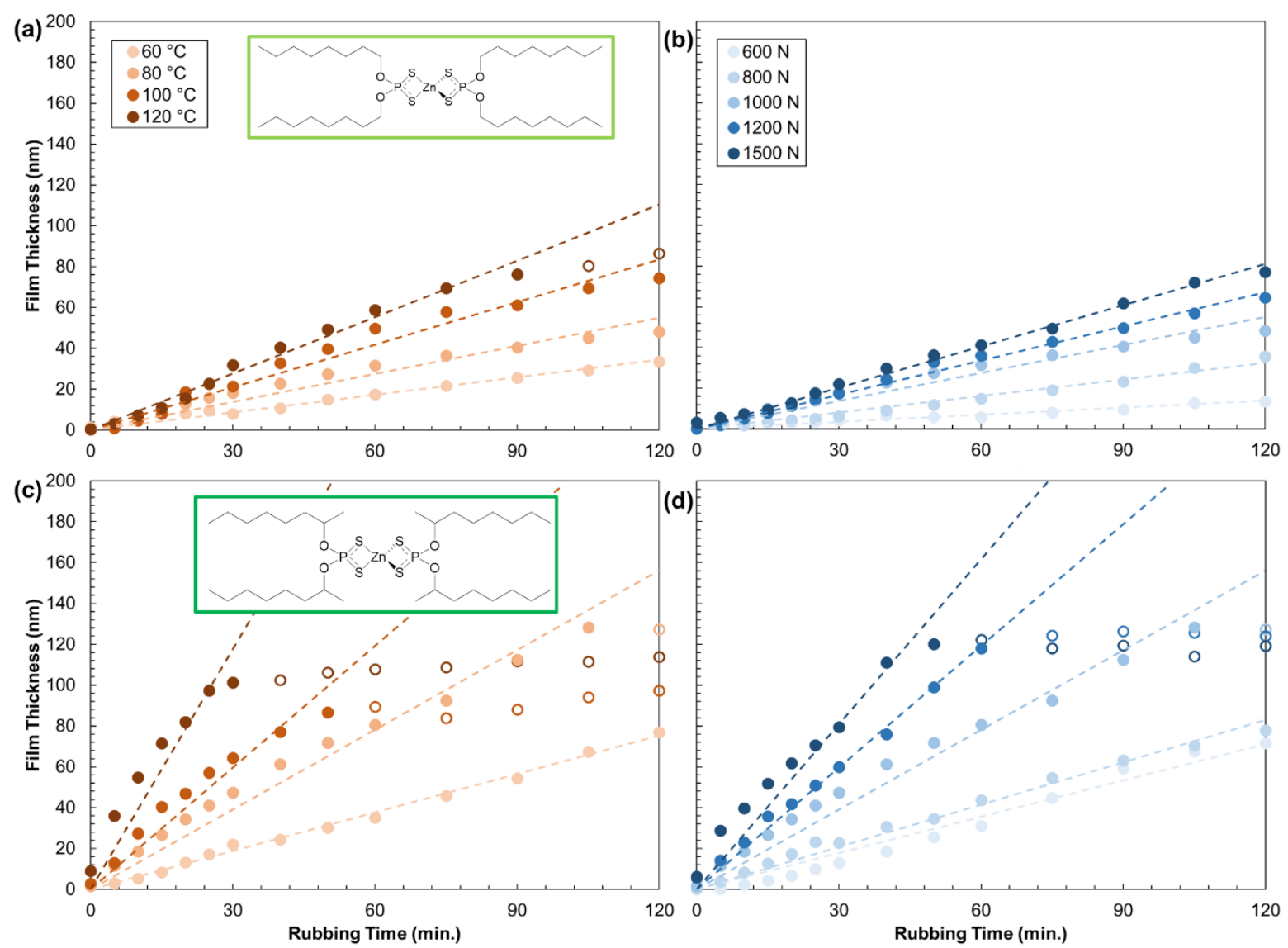

Figure 4. Variation in the tribofilm formation rate with temperature at a constant load of $1000 \mathrm{~N}$ $(a, c)$ and shear stress at a constant temperature of $80{ }^{\circ} \mathrm{C}(\mathrm{b}, \mathrm{d})$ for primary oct-1-yl $(\mathrm{a}, \mathrm{b})$ and secondary oct-2-yl (c, d) ZDDPs. Results for the other ZDDPs are shown in the Figure S2 and Figure S3. Dashed lines are the linear fits used to calculate the rates assuming zero-order kinetics; only the filled symbols are used for the fitting.

Figure 5 shows the temperature and shear stress dependence of the tribofilm growth rate for all the ZDDPs. The rates are calculated from the slopes of the linear fits in Figure 4, Figure S2, and Figure S3. For all the ZDDPs, the rate increases exponentially with both temperature and shear stress, which is indicative of a SATA process. ${ }^{11}$ Under all the conditions studied, the tribofilm growth rate is much faster for the secondary ZDDPs than the primary ZDDPs. ${ }^{16}$ The tribofilm growth rate 
is negligible $\left(<0.1 \mathrm{~nm} \mathrm{~min}^{-1}\right)$ for the dodec-1-yl ZDDP when $\mathrm{T}<80^{\circ} \mathrm{C}$, irrespective of the load. This could be due to the formation of a close-packed SAM by the dodec-1-yl ZDDP at low temperature that become less ordered (or possibly partially desorbed) at higher temperature, allowing additional ZDDP molecules to adsorb, decompose, and form tribofilms. For dodec-1-yl thiol SAMs on gold surfaces, the order-disorder transition temperature is $50{ }^{\circ} \mathrm{C},{ }^{57}$ while the desorption temperature is $110{ }^{\circ} \mathrm{C} .{ }^{59}$ The headgroup-substrate affinity will affect these values, so it is not possible to determine whether an order-disorder transition or desorption is the primary cause of the substantial increase in reactivity of the dodec-1-yl ZDDP above $80^{\circ} \mathrm{C}$. From an applicationfocused perspective, this type of antiwear additive could be very useful in high-temperature applications (such as heavy-duty diesel engines ${ }^{60}$ ) because it would effectively form tribofilms and also provide very low boundary friction under these conditions. ${ }^{9}$
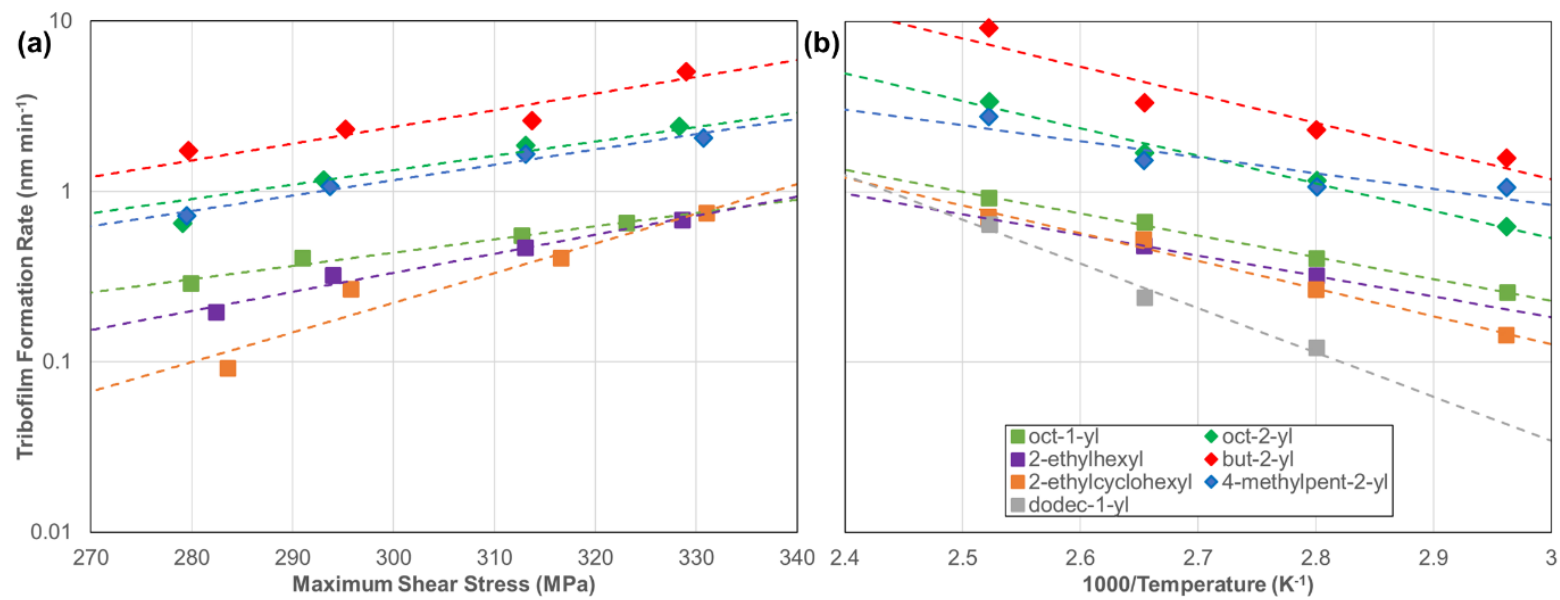

Figure 5. Change in the tribofilm growth rate for the various ZDDPs with (a) maximum shear stress, $\tau_{\max }$, at $80{ }^{\circ} \mathrm{C}$ and (b) inverse temperature, $1000 / T$, at $1000 \mathrm{~N}\left(P_{\max }=3 \mathrm{GPa}\right)$. Dashed lines are exponential fits of the data to Equation $1 .{ }^{13}$ The rate-shear stress data for the dodec-1-yl ZDDP is not shown since it was performed at a different temperature $\left(120^{\circ} \mathrm{C}\right)$. 
Using Equation $1,{ }^{13}$ the slopes of the rate-stress (Figure 5a) plots were used to calculate $\Delta V^{*}$, while the slopes and intercepts of the rate-temperature (Figure 5b) plots were used to calculate $E_{a}$ and $\ln (A)$, respectively (see Table 1 and Figure S4). ${ }^{61}$ The $\Delta V^{*}$ values are also normalized by the molecular volumes, $V_{\mathrm{mol}}$. The $V_{\mathrm{mol}}$ values shown in Table 1 were calculated using the $3 \mathrm{~V}$ software with a probe size of $3 \AA^{3}{ }^{62}$ The ZDDP structures used were relaxed in ChemBio3D Ultra 14 Suite (PerkinElmer, Waltham, MA) using the MM2 forcefield. ${ }^{63}$

The $\Delta V^{*}$ is slightly smaller for the primary oct-1-yl $\left(0.09 \mathrm{~nm}^{3}\right)$ ZDDP than the secondary oct-2-yl $\left(0.10 \mathrm{~nm}^{3}\right)$ ZDDP, implying a similar stress-dependency of their reactivity. The $E_{a}$ is higher for the oct-2-yl $\left(66 \mathrm{~kJ} \mathrm{~mol}^{-1}\right)$ than the oct-1-yl $\left(56 \mathrm{~kJ} \mathrm{~mol}^{-1}\right)$ ZDDP, despite the lower thermal stability of secondary ZDDPs. ${ }^{5,64}$ These $E_{a}$ values are consistent with previous measurements for commercial secondary $\left(82 \mathrm{~kJ} \mathrm{~mol}^{-1}\right),{ }^{16}$ primary $\left(56 \mathrm{~kJ} \mathrm{~mol}^{-1}\right),{ }^{16}$ and mixed primary/secondary $(79$ $\left.\mathrm{kJ} \mathrm{mol}^{-1}\right)^{10}$ ZDDPs. On the other hand, $A$ is around than two orders of magnitude larger for the oct2-yl $\left(2.5 \times 10^{5} \mathrm{~nm} \mathrm{~s}^{-1}\right)$ than the oct-1-yl $\left(5.9 \times 10^{3} \mathrm{~nm} \mathrm{~s}^{-1}\right)$ ZDDP. Since the difference in $E_{a}$ suggests faster rates for oct-1-yl than oct-2-yl, the experimentally observed larger rates for oct-2yl must be primarily due to the differences in $\Delta V^{*}$ and particularly $A$. This can be visualized in Figure 3, where the rates for the oct-2-yl ZDDP are shifted vertically (larger intercept) with respect to the oct-1-yl ZDDP. These observations are also consistent with the differences noted by Zhang et al. ${ }^{16}$, although the differences in $A$ between the commercial secondary $\left(2.0 \times 10^{7} \mathrm{~nm} \mathrm{~s}^{-1}\right)$ and primary $\left(1.1 \times 10^{3} \mathrm{~nm} \mathrm{~s}^{-1}\right)$ were even larger than in the current study. The current experiments support the hypothesis that the mechanochemical decomposition of primary and secondary ZDDPs proceeds through different chemical mechanisms. ${ }^{16}$ Indeed, previous glassware experiments ${ }^{5,64}$ and first principles calculations ${ }^{65}$ of thermal decomposition in the absence of steel surfaces have 
shown that $\mathrm{C}-\mathrm{O}$ bonds are broken through different mechanisms for primary and secondary ZDDPs; alkyl group transfer (from - OR to $-\mathrm{SR}$ ) for the former and alkene elimination for the latter.

Table 1. Kinetic parameters extracted from the fits to Equation 1 shown in Figure 3. Mean values are shown with $95 \%$ confidence intervals. $E_{a}$ and $A$ calculated at $1000 \mathrm{~N}, \Delta V^{*}$ calculated at $80{ }^{\circ} \mathrm{C}$ (*dodec-1-yl $\Delta V^{*}$ calculated at $120^{\circ} \mathrm{C}$ ).

\begin{tabular}{|l|l|l|l|l|l|l|}
\hline Substituent & $E_{a}$ & $A$ & $\ln (A)$ & $\Delta V^{*}$ & $V_{\mathrm{mol}}$ & $\Delta V^{*} / V_{\mathrm{mol}}$ \\
& \multirow{2}{*}{$\mathrm{kJ} \mathrm{mol}^{-1}$} & $\mathrm{~nm} \mathrm{~s}{ }^{-1}$ & & $\mathrm{~nm}^{3}$ & $\mathrm{~nm}^{3}$ & $\%$ \\
\hline oct-1-yl & $56 \pm 1$ & $5.9 \times 10^{3}$ & $9 \pm 1$ & $0.09 \pm 0.01$ & 1.26 & $7 \pm 1$ \\
\hline 2-ethylhexyl & $68 \pm 2$ & $1.9 \times 10^{4}$ & $10 \pm 1$ & $0.13 \pm 0.01$ & 1.32 & $10 \pm 1$ \\
\hline 2-cyclohexylethyl & $102 \pm 3$ & $2.1 \times 10^{7}$ & $17 \pm 1$ & $0.20 \pm 0.04$ & 1.18 & $17 \pm 3$ \\
\hline dodec-1-yl & $123 \pm 9$ & $1.8 \times 10^{10}$ & $24 \pm 3$ & $0.19 \pm 0.02^{*}$ & 1.72 & $11 \pm 1^{*}$ \\
\hline oct-2-yl & $66 \pm 4$ & $2.5 \times 10^{5}$ & $12 \pm 1$ & $0.10 \pm 0.02$ & 1.18 & $9 \pm 2$ \\
\hline but-2-yl & $72 \pm 7$ & $1.7 \times 10^{6}$ & $14 \pm 3$ & $0.11 \pm 0.02$ & 0.78 & $14 \pm 3$ \\
\hline 4-methylpent-2-yl & $55 \pm 6$ & $4.4 \times 10^{3}$ & $8 \pm 2$ & $0.10 \pm 0.02$ & 1.03 & $9 \pm 2$ \\
\hline
\end{tabular}

The $\Delta V^{*} / V_{\text {mol }}$ values in Table 1 suggest that a $7-17 \%$ deformation of the ZDDP molecules occurs when moving from the ground state to the transition state for the mechanochemical reaction. ${ }^{66}$ These values fall within the range estimated for other tribological processes involving molecular 
systems (2-27\%), which have been derived both from experiments and molecular simulations. ${ }^{14,61,67-70}$ The $\Delta V^{*}$ values are similar to those obtained previously for commercial primary $\left(0.13 \mathrm{~nm}^{3}\right)$ and secondary $\left(0.15 \mathrm{~nm}^{3}\right)$ ZDDPs. ${ }^{16}$ There do not seem to be any systematic differences between the $\Delta V^{*}$ values for primary and secondary ZDDPs. However, the primary $\mathrm{C}_{8}$ ZDDPs containing branched (2-ethylhexyl, $0.13 \mathrm{~nm}^{3}$ ) and particularly the bulky cycloaliphatic (2cyclohexylethyl, $0.20 \mathrm{~nm}^{3}$ ) substituents have larger $\Delta V^{*}$ values than those with linear (oct-1-yl, $0.09 \mathrm{~nm}^{3}$ ) alkyl groups. The exact physicochemical meaning of $\Delta V^{*}$ remains unclear for tribochemical reactions; $;^{71,72}$ however, a larger activation volume (or activation length ${ }^{21,22}$ ) implies that, at a given stress, there is a greater distortion of the molecule from the ground state to transition state along the reaction path. ${ }^{66}$ In other words, compared to the oct-1-yl groups, the 2-ethylhexyl and particularly 2-cyclohexylethyl groups act as metaphorical crowbars, allowing the shear (or tensile $\left.{ }^{13}\right)$ stress to be transmitted across a greater distance as the $\mathrm{C}-\mathrm{O}$ bond-breaking reaction proceeds. $^{25}$ This implies that the lever-arm effect, previously identified in polymer mechanochemistry under applied force, ${ }^{21}$ can also affect mechanochemical reactivity of comparatively small organometallic compounds such as ZDDP under applied stress. From a practical perspective, ZDDPs with branched and cycloaliphatic substituents could be particularly useful for wear protection in low-temperature, high-stress applications, such as gear oils. ${ }^{73}$ 


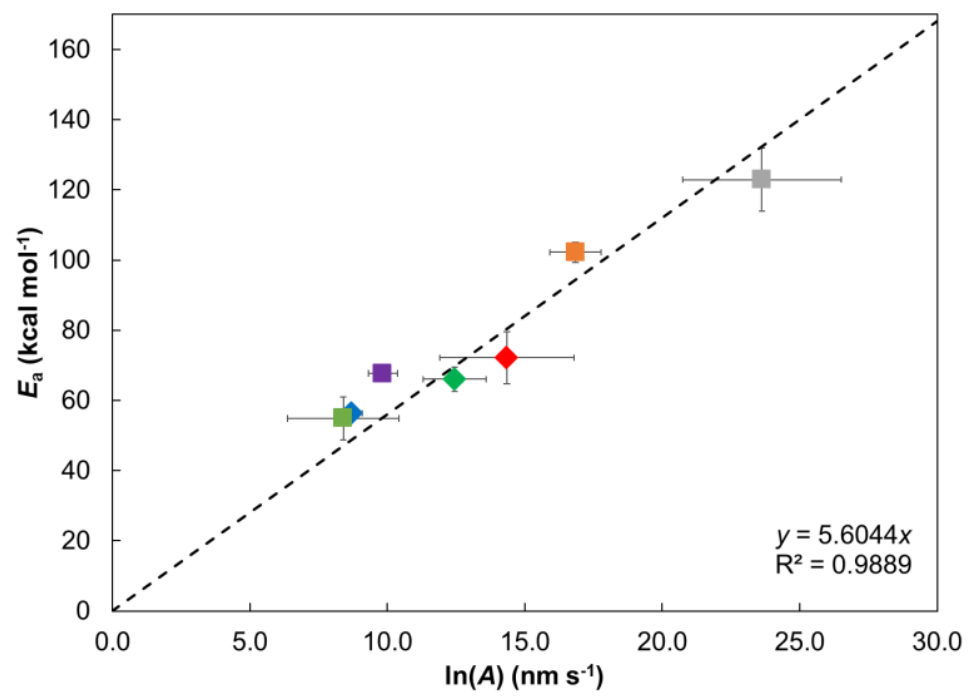

Figure 6. Linear relation between $E_{a}$ and $\ln (A)$ for the different ZDDPs studied, suggesting a kinetic compensation effect. ${ }^{74}$ Vertical and horizontal bars show $95 \%$ confidence intervals. Legend is the same as in Figure 5.

Figure 6 shows that, for the various ZDDPs studied here, there is a linear relationship between $E_{a}$ and $\ln (A)$, which is indicative of the kinetic compensation effect. ${ }^{74}$ ZDDPs that have a large $E_{a}$ also show higher $\ln (A)$ (e.g. dodec-1-yl), which suggests both a high energy barrier and a high frequency of successful collisions. ${ }^{12}$ From transition state theory, ${ }^{66}$ this can also be interpreted as the tribofilm growth reactions having a larger enthalpic barrier, but also a smaller entropic penalty. ${ }^{74}$ ZDDPs with primary linear alkyl groups sit on the linear fit in Figure 6 (within statistical uncertainty), while those with branched (2-ethylhexyl) and cycloaliphatic (2-ethylcylohexyl) primary alkyl groups sit above it. Thus, ZDDPs with branched and cycloaliphatic groups have a larger $E_{a}$ for a given $\ln (A)$ compared to ZDDPs with linear alkyl groups, which can be attributed to their higher thermal stability due to increased steric bulk. Conversely, most of the ZDDPs with secondary alkyl groups (oct-2-yl and but-2-yl) fall below the linear fit in Figure 6, implying a 
smaller $E_{a}$ for a given $\ln (A)$, which can be attributed to their lower thermal stability, due to the increased stability of the secondary carbocation. ${ }^{5,64}$

In this study, we have demonstrated that the Bell model ${ }^{13}$ can be used to rationalize the large differences in mechanochemical reactivity of the various ZDDPs studied (Figure S5). Linear secondary alkyl ZDDPs are inherently less stable than primary ones with the same chain length under mechanochemical conditions, which is mostly due to a higher pre-exponential factor (Table 1). ${ }^{16,61}$ Since they form SAMs with a higher packing density, ZDDPs with longer chain lengths require higher temperatures to form tribofilms, which manifests as a higher activation energy. We observe an increase in the activation volume moving from linear, to branched, and to cyloaliphatic substituents, which suggests a molecular-scale analogue of the lever-arm effect that has been observed in polymer mechanochemistry. ${ }^{21}$ These kinetic parameters can be directly used to inform macroscale models of tribofilm growth inside rubbing contacts. ${ }^{35-38}$ We also expect that, coupled with information regarding their friction and wear performance under mixed/boundary conditions, ${ }^{9}$ the improved understanding of substituents effects on tribofilm growth identified here under mechanochemical conditions to be useful in the rational design of antiwear additives with improved performance for specific applications such as heavy-duty diesel engines ${ }^{60}$ and gear oils. ${ }^{73}$

\section{CONCLUSIONS}

We have studied the tribofilm formation rate of four primary and three secondary ZDDPs with well-defined alkyl substituents in steel/steel contacts under full-film EHL conditions. For all of the ZDDPs, the tribofilm growth rate increases exponentially with temperature and shear stress, which confirms that this is a SATA process. We have shown how the parameters for the Bell equation can be used to rationalize the order of mechanochemical reactivity for the different ZDDPs. The 
chain-length, branching and presence of cycloaliphatic groups in the ZDDP alkyl substituents affects the packing density, steric hindrance, and stress transmission, which leads to significant differences in the activation energy, pre-exponential factor, and activation volume. We anticipate that these results will be useful both for the rational design of lubricant additives with improved performance and the development of more accurate macroscale tribology models that consider the effect of growing of tribofilms.

\section{ASSOCIATED CONTENT}

Supporting Information. Additional figures showing: a schematic of the ETM-SLIM setup, additional tribofilm growth measurements, bar charts to visualize the Bell model parameters for the various ZDDPs, and a plot comparing the rates measured experimentally to those predicted from the Bell model.

\section{AUTHOR INFORMATION}

The authors declare no competing financial interests.

\section{ACKNOWLEDGMENTS}

We thank the Engineering and Physical Sciences Research Council (EPSRC) for funding via grant EP/P030211/1. J.Z. thanks Shell for funding through the Shell-Imperial University Technology Centre for Fuels \& Lubricants. J.P.E was supported by the Royal Academy of Engineering through the Research Fellowships scheme. We thank PCS Instruments Ltd. for provision of the ETM tribometer, INEOS Group Ltd. for the supply of base oils, and Afton Chemical Corp. for the supply of ZDDP additives. We thank Carlos Ayestarán Latorre (Imperial College London) and Joseph E. Remias (Afton Chemical) for useful discussions. 


\section{REFERENCES}

(1) Liu, S.; Josephson, T. R.; Athaley, A.; Chen, Q. P.; Norton, A.; Ierapetritou, M.; Siepmann, J. I.; Saha, B.; Vlachos, D. G. Renewable Lubricants with Tailored Molecular Architecture. Science Advances 2019, 5, eaav5487.

(2) Taylor, R. I. Tribology and Energy Efficiency: From Molecules to Lubricated Contacts to Complete Machines. Faraday Discussions 2012, 156, 361-382.

(3) Spikes, H. The History and Mechanisms of ZDDP. Tribology Letters 2004, 17, 469-489.

(4) Spikes, H. Low- and Zero-Sulphated Ash, Phosphorus and Sulphur Anti-Wear Additives for Engine Oils. Lubrication Science 2008, 20, 103-136.

(5) Jones, R. B.; Coy, R. C. The Chemistry of the Thermal Degradation of Zinc Dialkyldithiophosphate Additives. ASLE Transactions 1981, 24, 91-97.

(6) Mosey, N. J.; Woo, T. K. An Ab Initio Molecular Dynamics and Density Functional Theory Study of the Formation of Phosphate Chains from Metathiophosphates. Inorganic Chemistry 2006, 45, 389-395.

(7) Graham, J. F.; McCague, C.; Norton, P. R. Topography and Nanomechanical Properties of Tribochemical Films Derived from Zinc Dialkyl and Diaryl Dithiophosphates. Tribology Letters 1999, 6, 149-157.

(8) Fujita, H.; Spikes, H. A. The Formation of Zinc Dithiophosphate Antiwear Films. Proceedings of the Institution of Mechanical Engineers, Part J 2004, 218, 265-277.

(9) Zhang, J.; Ueda, M.; Campen, S.; Spikes, H. Boundary Friction of ZDDP Tribofilms. Tribology Letters 2021, 69, 8.

(10) Gosvami, N. N.; Bares, J. A.; Mangolini, F.; Konicek, A. R.; Yablon, D. G.; Carpick, R. W. Mechanisms of Antiwear Tribofilm Growth Revealed in Situ by Single-Asperity Sliding Contacts. Science. 2015, 348, 102-106.

(11) Spikes, H. A. Stress-Augmented Thermal Activation: Tribology Feels the Force. Friction 2018, 6, 1-31.

(12) Evans, M. G.; Polanyi, M. Some Applications of the Transition State Method to the Calculation of Reaction Velocities, Especially in Solution. Transactions of the Faraday Society 1935, 31, 875-894.

(13) Bell, G. I. Models for the Specific Adhesion of Cells to Cells. Science. 1978, 200, 618-627.

(14) Boscoboinik, A.; Olson, D.; Adams, H.; Hopper, N.; Tysoe, W. T. Measuring and Modelling Mechanochemical Reaction Kinetics. Chemical Communications 2020, 56, 7730 .

(15) Zhang, J.; Spikes, H. On the Mechanism of ZDDP Antiwear Film Formation. Tribology Letters 2016, 63, 24. 
(16) Zhang, J.; Ewen, J. P.; Ueda, M.; Wong, J. S. S.; Spikes, H. A. Mechanochemistry of Zinc Dialkyldithiophosphate on Steel Surfaces under Elastohydrodynamic Lubrication Conditions. ACS Applied Materials \& Interfaces 2020, 12, 6662-6676.

(17) Dorgham, A.; Parsaeian, P.; Azam, A.; Wang, C.; Morina, A.; Neville, A. Single-Asperity Study of the Reaction Kinetics of P-Based Triboreactive Films. Tribology International 2019, 133, 288-296.

(18) Hanneman, W. W.; Porter, R. S. The Thermal Decomposition of Dialkyl Phosphates and O,O-Dialkyl Dithiophosphates. The Journal of Organic Chemistry 1964, 29, 2996-2998.

(19) Odell, J. A.; Keller, A. Flow-induced Chain Fracture of Isolated Linear Macromolecules in Solution. Journal of Polymer Science Part B: Polymer Physics 1986, 24, 1889-1916.

(20) Oka, H.; Imato, K.; Sato, T.; Ohishi, T.; Goseki, R.; Otsuka, H. Enhancing Mechanochemical Activation in the Bulk State by Designing Polymer Architectures. ACS Macro Letters 2016, 5, 1124-1127.

(21) Klukovich, H. M.; Kouznetsova, T. B.; Kean, Z. S.; Lenhardt, J. M.; Craig, S. L. A Backbone Lever-Arm Effect Enhances Polymer Mechanochemistry. Nature Chemistry 2013, 5, 110-114.

(22) Wang, J.; Kouznetsova, T. B.; Kean, Z. S.; Fan, L.; Mar, B. D.; Martínez, T. J.; Craig, S. L. A Remote Stereochemical Lever Arm Effect in Polymer Mechanochemistry. Journal of the American Chemical Society 2014, 136, 15162-15165.

(23) Brown, C. L.; Bowser, B. H.; Meisner, J.; Kouznetsova, T. B.; Seritan, S.; Martinez, T. J.; Craig, S. L. Substituent Effects in Mechanochemical Allowed and Forbidden Cyclobutene Ring-Opening Reactions. Journal of the American Chemical Society 2021, 143, 3846-3855.

(24) Tian, Y.; Boulatov, R. Quantum-Chemical Validation of the Local Assumption of Chemomechanics for a Unimolecular Reaction. ChemPhysChem 2012, 13, 2277-2281.

(25) Bowser, B. H.; Wang, S.; Kouznetsova, T. B.; Beech, H. K.; Olsen, B. D.; Rubinstein, M.; Craig, S. L. Single-Event Spectroscopy and Unravelling Kinetics of Covalent Domains Based on Cyclobutane Mechanophores. Journal of the American Chemical Society 2021, 143, 5269-5276.

(26) Wells, P. R. Linear Free Energy Relationships. Chemical Reviews 1963, 63, 171-219.

(27) Barbee, M. H.; Kouznetsova, T.; Barrett, S. L.; Gossweiler, G. R.; Lin, Y.; Rastogi, S. K.; Brittain, W. J.; Craig, S. L. Substituent Effects and Mechanism in a Mechanochemical Reaction. Journal of the American Chemical Society 2018, 140, 12746-12750.

(28) Krupička, M.; Marx, D. Disfavoring Mechanochemical Reactions by Stress-Induced Steric Hindrance. Journal of Chemical Theory and Computation 2015, 11, 841-846.

(29) Fernández-González, M. Á.; Frutos, L. M. The Concept of Substituent-Induced Force in the Rationale of Substituent Effect. The Journal of Chemical Physics 2021, 154, 224106. 
(30) Spikes, H.; Jie, Z. History, Origins and Prediction of Elastohydrodynamic Friction. Tribology Letters 2014, 56, 1-25.

(31) Uzarevic, K.; Halasz, I.; Frisic, T. Real-Time and In Situ Monitoring of Mechanochemical Reactions: A New Playground for All Chemists. The Journal of Physical Chemistry Letters 2015, 6, 4129-4140.

(32) Ueda, M.; Kadiric, A.; Spikes, H. A. On the Crystallinity and Durability of ZDDP Tribofilm. Tribology Letters 2019, 67, 123.

(33) Fischer, T. E. Tribochemistry. Annual Reviews of Materials Science 1988, 18, 303-323.

(34) Ueda, M.; Kadiric, A.; Spikes, H. ZDDP Tribofilm Formation on Non-Ferrous Surfaces. Tribology Online 2020, 15, 318-331.

(35) Ghanbarzadeh, A.; Parsaeian, P.; Morina, A.; Wilson, M. C. T.; van Eijk, M. C. P.; Nedelcu, I.; Dowson, D.; Neville, A. A Semi-Deterministic Wear Model Considering the Effect of Zinc Dialkyl Dithiophosphate Tribofilm. Tribology Letters 2016, 61, 12.

(36) Akchurin, A.; Bosman, R. A Deterministic Stress-Activated Model for Tribo-Film Growth and Wear Simulation. Tribology Letters 2017, 65, 59.

(37) Chen, Z.; Gu, C.; Tian, T. Modeling of Formation and Removal of ZDDP Tribofilm on Rough Surfaces. Tribology Letters 2021, 69, 13.

(38) Yang, J.; Pu, W.; Pei, X. A Deterministic Tribofilm Growth Model Considering Flash Temperature and Surface Roughness in Mixed Lubrication. Surface Topography: Metrology and Properties 2021, 9, 025017.

(39) Guo, Y. B.; Liu, C. R. Mechanical Properties of Hardened AISI 52100 Steel in Hard Machining Processes. Journal of Manufacturing Science and Engineering, Transactions of the ASME 2002, 124, 1-9.

(40) Menzer, S.; Phillips, J. R.; Slawin, A. M. Z.; Williams, D. J.; Woollins, J. D. Structural Characterisation of Basic Zinc O,O'-Dialkyl Dithiophosphosphate and Two Isomeric Examples of Zinc Monothiophosphates. Journal of the Chemical Society, Dalton Transactions 2000, 3269-3273.

(41) Plaza, S. The Adsorption of Zinc Dibutyldithiophosphates on Iron and Iron Oxide Powders. ASLE Transactions 1987, 30, 233-240.

(42) Elimelech, O.; Aviv, O.; Oded, M.; Peng, X.; Harries, D.; Banin, U. Entropy of Branching Out: Linear versus Branched Alkylthiols Ligands on CdSe Nanocrystals. ACS Nano 2022, acsnano.1c10430.

(43) Kwon, S.; Choi, Y.; Choi, J.; Kang, H.; Chung, H.; Noh, J. Fast Displacement and Structural Transition of Cyclohexanethiol Self-Assembled Monolayers by Octanethiols on Au(111). Ultramicroscopy 2008, 108, 1311-1314. 
(44) Gohar, R.; Cameron, A. Optical Measurement of Film Thickness Under ElastoHydrodynamic Lubrication. Nature 1963, 200, 458-459.

(45) Chittenden, R. J.; Dowson, D.; Dunn, J. F.; Taylor, C. M. A Theoretical Analysis of the Isothermal Elastohydrodynamic Lubrication of Concentrated Contacts I. Direction of Lubricant Entrainment Coincident with the Major Axis of the Hertzian Contact Ellipse. Proceedings of the Royal Society of London, Series A 1985, 397, 245-269.

(46) Evans, C. R.; Johnson, K. L. The Influence of Surface Roughness on Elastohydrodynamic Traction. Proceedings of the Institution of Mechanical Engineers, Part C 1987, 201, 145150.

(47) Bair, S. High-Pressure, High-Shear Stress Rheology of a Polybutene. Journal of NonNewtonian Fluid Mechanics 2001, 97, 53-65.

(48) Archard, J. F. The Temperature of Rubbing Surfaces. Wear 1959, 2, 438-455.

(49) Reddyhoff, T.; Schmidt, A.; Spikes, H. Thermal Conductivity and Flash Temperature. Tribology Letters 2019, 67, 22.

(50) Cann, P. M.; Spikes, H. A.; Hutchinson, J. The Development of a Spacer Layer Imaging Method (SLIM) for Mapping Elastohydrodynamic Contacts. Tribology Transactions 1996, 39, 915-921.

(51) Aoki, S.; Suzuki, A.; Masuko, M. Comparison of Sliding Speed Dependency of Friction between Steel Surfaces Lubricated with Several ZnDTPs with Different Hydrocarbon Moieties. Proceedings of the Institution of Mechanical Engineers, Part J 2006, 220, 343351.

(52) Jiang, S. Y.; Frazier, R.; Yamaguchi, E. S.; Blanco, M.; Dasgupta, S.; Zhou, Y. H.; Cagin, T.; Tang, Y. C.; Goddard III, W. A. The SAM Model for Wear Inhibitor Performance of Dithiophosphates on Iron Oxide. The Journal of Physical Chemistry B 1997, 101, 77027709.

(53) Zhou, Y. H.; Jiang, S. Y.; Cagin, T.; Yamaguchi, E. S.; Frazier, R.; Ho, A.; Tang, Y. C.; Goddard III, W. A. Application of the Self-Assembled Monolayer (SAM) Model to Dithiophosphate and Dithiocarbamate Engine Wear Inhibitors. The Journal of Physical Chemistry A 2000, 104, 2508-2524.

(54) Zamborini, F. P.; Crooks, R. M. Corrosion Passivation of Gold by N-Alkanethiol SelfAssembled Monolayers: Effect of Chain Length and End Group. Langmuir 1998, 14, 32793286.

(55) Templeton, A. C.; Hostetler, M. J.; Kraft, C. T.; Murray, R. W. Reactivity of MonolayerProtected Gold Cluster Molecules: Steric Effects. Journal of the American Chemical Society 1998, 120, 1906-1911.

(56) Dordi, B.; Schönherr, H.; Vancso, G. J. Reactivity in the Confinement of Self-Assembled Monolayers: Chain Length Effects on the Hydrolysis of N-Hydroxysuccinimide Ester Disulfides on Gold. Langmuir 2003, 19, 5780-5786. 
(57) Fenter, P.; Eisenberger, P.; Liang, K. S. Chain-Length Dependence of the Structures and Phases of CH3(CH2)n-1 SH Self-Assembled on Au(111). Physical Review Letters 1993, 70, 2447-2450.

(58) Bin, X.; Mischki, T. K.; Fan, C.; Lopinski, G. P.; Wayner, D. D. M. Electrochemical Characterization of Si(111) Modified with Linear and Branched Alkyl Chains. The Journal of Physical Chemistry C 2007, 111, 13547-13553.

(59) Pi, U. H.; Kim, J. H.; Yu, H. Y.; Park, C. W.; Choi, S. Y.; Kim, Y. K.; Ha, J. S. Enhanced Surface Evolution Induced by the Molecular Desorption in Dodecanethiol Self-Assembled Monolayer on Au(1 1 1). Surface Science 2006, 600, 625-631.

(60) Pierce, D.; Haynes, A.; Hughes, J.; Graves, R.; Maziasz, P.; Muralidharan, G.; Shyam, A.; Wang, B.; England, R.; Daniel, C. High Temperature Materials for Heavy Duty Diesel Engines: Historical and Future Trends. Progress in Materials Science 2019, 103, 109-179.

(61) Ayestarán Latorre, C.; Remias, J. E.; Moore, J. D.; Spikes, H. A.; Dini, D.; Ewen, J. P. Mechanochemistry of Phosphate Esters Confined between Sliding Iron Surfaces. Communications Chemistry 2021, 4, 178.

(62) Voss, N. R.; Gerstein, M. 3V: Cavity, Channel and Cleft Volume Calculator and Extractor. Nucleic Acids Research 2010, 38, 555-562.

(63) Allinger, N. L. Conformational Analysis. 130. MM2. A Hydrocarbon Force Field Utilizing V1 and V2 Torsional Terms. Journal of the American Chemical Society 1977, 99, 81278134.

(64) Dickert, J. J.; Rowe, C. N. The Thermal Decomposition of Metal O,ODialkylphosphorodithioates. The Journal of Organic Chemistry 1967, 32, 647-653.

(65) Mosey, N. J.; Woo, T. K. A Quantum Chemical Study of the Unimolecular Decomposition Mechanisms of Zinc Dialkyldithiophosphate Antiwear Additives. The Journal of Physical Chemistry A 2004, 108, 6001-6016.

(66) Eyring, H. The Activated Complex in Chemical Reactions. The Journal of Chemical Physics 1935, 3, 107.

(67) Yeon, J.; He, X.; Martini, A.; Kim, S. H. Mechanochemistry at Solid Surfaces: Polymerization of Adsorbed Molecules by Mechanical Shear at Tribological Interfaces. ACS Applied Materials and Interfaces 2017, 9, 3142-3148.

(68) He, X.; Kim, S. H. Mechanochemistry of Physisorbed Molecules at Tribological Interfaces: Molecular Structure Dependence of Tribochemical Polymerization. Langmuir 2017, 33, 2717-2724.

(69) Khajeh, A.; He, X.; Yeon, J.; Kim, S. H.; Martini, A. Mechanochemical Association Reaction of Interfacial Molecules Driven by Shear. Langmuir 2018, 34, 5971-5977.

(70) Chen, X.; Kawai, K.; Zhang, H.; Fukuzawa, K.; Koga, N.; Itoh, S.; Azuma, N. ReaxFF Reactive Molecular Dynamics Simulations of Mechano-Chemical Decomposition of 
Perfluoropolyether Lubricants in Heat-Assisted Magnetic Recording. The Journal of Physical Chemistry C 2020, 124, 22496-22505.

(71) Li, Z.; Szlufarska, I. Physical Origin of the Mechanochemical Coupling at Interfaces. Physical Review Letters 2021, 126, 076001.

(72) Martini, A.; Kim, S. H. Activation Volume in Shear-Driven Chemical Reactions. Tribology Letters 2021, 69, 150.

(73) Adebogun, A.; Hudson, R.; Matthews, A.; Withers, P. J. Industrial Gear Oils: Influence of Bulk Oil Temperature and Contact Pressure on Tribological Performance and Subsurface Changes. Tribology Letters 2020, 68, 48.

(74) Roduner, E. Understanding Catalysis. Chemical Society Reviews 2014, 43, 8226-8239.

\section{TOC GRAPHIC}

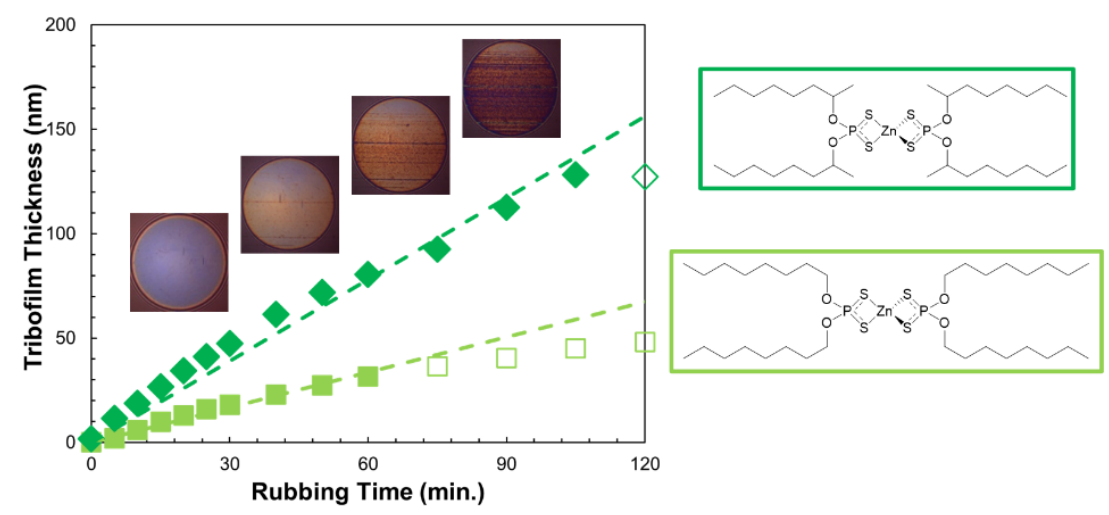

